\title{
Validación del cuestionario de hábitos de vida (LIFE-H) para evaluar la participación de niños/as y jóvenes con Parálisis Cerebral en Chile
}

\author{
Validation of Life Habits Assessment Questionnaire (LIFE-H) to assess \\ participation of children and young people with Cerebral Palsy in Chile
}

\author{
Claudia P. Pérez-Salas \\ Rubia Cobo-Rendón \\ Departamento de Psicología, Universidad de Concepción, Chile \\ Constanza Herrera \\ Departamento de Educación, Facultad de Humanidades, Universidad de Santiago de Chile. \\ Claudio Bustos \\ Departamento de Psicología, Universidad de Concepción, Chile \\ Jessica Candia \\ Departamento de Psiquiatría, Universidad de Concepción, Chile
}

Rec (21 de diciembre de 2018) Acept (14 de junio de 2019)

\begin{abstract}
Resumen
La participación es un derecho humano que debe ser garantizado. No obstante, variadas restricciones físicas y sociales dificultan el involucramiento de las personas con condiciones neurológicas en su comunidad. Muchos países de Latinoamérica no cuentan con instrumentos validados que permitan analizar la participación de estos grupos. Por esta razón, a través de un diseño instrumental esta investigación evalúa las propiedades psicométricas del cuestionario de hábitos de vida (LIFE-H) en una muestra de 123 personas con parálisis cerebral entre 9 y 21 años $(M=14.14 ; D E=2.89)$. Se detectaron adecuados índices de consistencia interna. No se replicó la estructura de dos dimensiones mediante análisis factorial confirmatorio. Sin embargo, el análisis factorial exploratorio de los datos evidenció un factor general que explicó el $69.55 \%$ de la varianza. Se concluye que el LIFE-H es un instrumento idóneo para el estudio de la participación como un constructo global en esta población.

Palabras clave: Validación, Participación, LIFE-H, Calidad de vida, Parálisis Cerebral.
\end{abstract}

Correspondencia: Claudia Perez-Salas, Departamento de Psicología, Universidad de Concepción, Chile, Fono +56-41-2204301, e-mail: cperezs@udec.cl

Este artículo contó con financiamiento CONICYT. Proyecto Fondecyt de Iniciación 11110358: El rol moderador de la participación sobre el funcionamiento cognitivo de escolares con Parálisis Cerebral. 


\begin{abstract}
Participation is a human right that must be guaranteed. However, various physical and social restrictions make it difficult for people with neurological conditions to participate in their community. Many Latin American countries do not have validated instruments to analyze the participation of these groups. The present investigation used an instrumental design to evaluate the psychometric properties of the Life Habits Assessment Questionnaire (LIFE-H) in a sample of 123 people with cerebral palsy between 9 and 21 years old $(\mathrm{M}=14.14 ; \mathrm{DE}=2.89)$. Adequate internal consistency indices were detected. The two-dimensional structure proposed by the authors was not replicated through confirmatory factor analysis. However, the exploratory factor analysis of the data evidenced a general factor that explained $69.55 \%$ of the variance. It is concluded that LIFE-H is an ideal instrument for the study of participation as a global construct in this population.

Key words: Validity, Participation, LIFE-H, Quality of life, Cerebral Palsy.
\end{abstract}

\title{
Introducción
}

La participación, es un concepto que ha cobrado importancia para la comprensión de la discapacidad desde su inclusión en la Clasificación Internacional del Funcionamiento, de la discapacidad y de la salud (CIF) presentado por la Organización Mundial de la Salud. En este esquema, se entiende a la participación como el involucramiento de una persona en situaciones de la vida diaria y se le considera como uno de los elementos centrales a la hora de evaluar su estado general de salud (Clasificación Internacional del Funcionamiento, de la discapacidad y de la salud, 2001).

La CIF concibe la participación como el resultado de la condición de salud de la persona y de los factores del ambiente en el que se desenvuelve. Así, uno de los componentes de la discapacidad es la restricción de la participación, la que a su vez es resultado de la interacción entre la condición de salud, la estructura, función corporal, factores personales y ambientales (Clasificación Internacional del Funcionamiento, de la discapacidad y de la salud, 2001). La discapacidad en este modelo, es comprendida desde una mirada multidimensional, siendo la participación de las personas el medio para la comprensión de esta y para el establecimiento de mecanismos que la incorporen dentro de los proceso de rehabilitación (Nahuelhual, Giaconi, \& Machuca, 2018).

La importancia de la participación radica en que promueve la salud y el bienestar del ser humano en todos los momentos de su vida. En primera instancia provee del contexto para que los niños y jóvenes se desarrollen y preparen para las transiciones de la vida (Piskur et al., 2014). Para la población infantil, la participación es un aspecto esencial en el desarrollo de habilidades, competencias para la vida y para el fortalecimiento de su salud física y mental (Nahuelhual et al., 2018); para las personas mayores, se considera como un factor determinante del éxito y envejecimiento saludable (Piskur et al., 2014).

En el caso de las personas con condiciones neurológicas, una adecuada participación es un factor que está relacionado con su calidad de vida y salud (Levasseur, Desrosiers \& Noreau, 2004; Levasseur, Desrosiers, \& St-Cyr Tribble, 2008). Esta participación puede ser realizada con ayuda humana o asistencia técnica, lo importante es que aun cuando se está recibiendo ayuda o asistencia, una persona con una condición neurológica pueda sentirse involucrada e inmersa en una actividad y ser parte de una comunidad (Anaby \& Law, 2013).

Se ha reportado, no obstante, que las personas con condiciones neurológicas como la Parálisis Cerebral (PC) presentan menores oportunidades educacionales y laborales que sus pares sin discapacidad (Michelsen, Uldall, Kejs, \& Madsen, 2005), además presentan una menor participación tanto en actividades formales (como asistir al colegio o realizar algún deporte) como no formales (actividades sin planificación como jugar, pasear, actividades de ocio) y tienen menores instancias de participación activa en la comunidad (Michelsen et al., 2014).

Dada la importancia de esta temática, se han presentado revisiones de la literatura con el objetivo de describir los más de 15 instrumentos que miden la participación en niños y adolescentes según planteamientos de organismos internaciones (e.g LIFE-H, ASKP, CHORES, CAPE Y PAC, CASP, PICO-Q, APS, CPQ, PEN-CY, QYPP, CEDL y C-PEM) (Chien, Rodger, Copley, \& Skorka, 2014; Nahuelhual et al., 2018). Los autores de estas revisiones refieren la existencia de diversas propiedades psicométricas, características en las escalas evaluadas e idiomas, debido a los distintos lugares donde fueron construidas (Canadá, Estados Unidos, e Israel), lo que amerita la realización 
de las respectivas traducciones, adaptaciones culturales y estimaciones de sus propiedades psicométricas para sus distintos usos en otros contextos (Chien et al., 2014; Nahuelhual et al., 2018).

De acuerdo a nuestro conocimiento, hay carencia de cuestionarios en habla hispana que midan la participación tal y como ha sido definido por la OMS. La escasez de instrumentos validados en español dificulta considerar esta variable como parte integrante de las investigaciones acerca de la inclusión social y/o el desarrollo integral de personas con discapacidad en nuestro contexto. El Assessment of Life Habits (LIFE-H) (Fougeyrollas et al., 1998) es uno de los instrumentos que cuentan con reconocimiento a nivel internacional, mayor confiabilidad y validez, para evaluar la participación de personas en situación de discapacidad (Morris, 2007) y según Chien et al (2014) es un buen instrumento para evaluar participación tal y como es entendida en la CIF, razón por la cual será foco de este estudio.

\section{Utilidad del LIFE-H en la evaluación de la participación}

El cuestionario de los hábitos de vida (LIFE-H) es un instrumento que ha sido objeto de numerosos estudios psicométricos originando diferentes versiones (Chien et al., 2014; Ziviani, Desha, Feeney, \& Boyd, 2012). Su objetivo es evaluar la participación de personas con discapacidad. Este es un cuestionario diseñado de forma flexible para ser completado directamente por niños, jóvenes, adultos o sus cuidadores, o puede ser completado por medio de una entrevista.

Está constituido por 12 indicadores vinculados a las actividades de vida la diaria (comunicación, cuidado personal, vivienda, desplazamiento, nutrición y condición física) y al desempeño en los roles sociales (recreación, responsabilidad, educación, vida en comunidad, trabajo y relaciones interpersonales) (Figueiredo, KornerBitensky, Rochette, \& Desrosiers, 2010; Fougeyrollas et al., 1998). Cubriendo siete de los nueve dominios propuestos por la CIF (2001). Debido a las diferencias en las descripciones de las actividades según el ciclo vital, el LIFE-H presenta dos formatos, uno para niños (5-13 años) y otro para jóvenes/ adultos(14-99 años). Este instrumento es empleado en investigación y como herramienta para evaluación clínica (Chien et al., 2014).

El LIFE-H fue creado en 1997, con 298 ítems (Fougeyrollas et al., 1998). Los primeros análisis psicométricos fueron realizados con dos versiones del instrumento (original y abreviada) en niños y adultos que sufrían lesión medular. Los resultados en su primera versión reportaron altos índices de consistencia interna en ambos grupos (versión abreviada $\alpha \geq .82$; versión original $\alpha \geq .90$ ). Así como adecuados niveles de correlación intra- clase

(ICC $=.80$ en el caso de los niños; ICC $=.89$ en el caso de los adultos) (Fougeyrollas et al., 1998).

El formato de niños, contiene 69 ítems en su forma corta y 242 en su forma general. Se elaboró tras un estudio de la validez de contenido en el cual participaron 12 expertos en áreas de rehabilitación y salud. Posteriormente, tras otra revisión del instrumento, se elaboró la versión 3.0, la cual cuenta con 240 ítems en su versión larga, y 69 ítems en su versión corta. En el año 2001 se elabora la versión 3.1 la cual incluye dos categorías de hábitos: las actividades diarias y los roles sociales (Noreau et al., 2004; Poulin \& Desrosiers, 2009). En todos los casos se mantienen los 12 indicadores que lo componen, variando el nivel de ítems que las constituyen.

El uso destacado del LIFE-H ha permitido la presentación de estudios que integran sus aportes. Por ejemplo, la literatura refiere una revisión sistemática sobre las propiedades psicométricas específicas del LIFE-Hen pacientes adultos con accidente cerebrovascular. Reportan en sus resultados identificar 11 estudios que cumplían con las características de selección, donde la confiabilidad test-retest fue considerada como excelente (coeficientes de correlación intraclase de 0.80 a 0.95 ). La confiabilidad inter-evaluadores estuvo en categorías de adecuada a excelente $(\mathrm{r}=0.64-0.91)$ y la validez convergente entre LIFE-H y medidas de independencia funcional fue adecuada a excelente $(r=0.57-0.91)$ (Figueiredo et al., 2010).

A nivel internacional y en el contexto infantil, el desarrollo de estudios psicométricos para el LIFE-H ha tenido desarrollo en los últimos años, esto debido a la necesidad de medir la participación de las actividades de los niños y por ser considerado como uno de los instrumentos más completo en la medición de los dominios de participación propuestos la CIF (Ziviani et al., 2012).

En el 2005 se presenta una revisión sistemática para identificar los instrumentos que midieran de forma adecuada la participación de niños con parálisis cerebral. En sus resultados, los autores refieren que la literatura presenta relativamente pocos instrumentos apropiados para medir la participación de los niños (solo siete fueron 
presentó adecuados niveles de confiabilidad entre las respuestas de los niños y sus padres, sin embargo para ese momento los autores refieren que se desconoce la fiabilidad especifica del niño o la familia (Morris, Kurinczuk, \& Fitzpatrick, 2005).

Posteriormente en el 2007, se examinaron las propiedades psicométricas LIFE-H para niños de 5 a 13 años de edad con diversas condiciones neurológicas (parálisis cerebral, mielomeningocele, neuropatía sensitivo-motora, lesión cerebral traumática y retraso en el desarrollo). La validez del contenido fue verificada por un panel de expertos de 29 personas, formado por padres, pediatras e investigadores. En este caso el LIFE-H mostró una alta confiabilidad con valores del coeficiente de correlación intra-clase de 0,78 o más para las 10 de las 11 categorías (Noreau et al., 2007).

Por su parte, Ziviani, Desha, Feeney \& Boyd, (2012) presentan una revisión sistemática que buscó examinar las propiedades psicométricas de instrumentos que miden la participación de niños con daño cerebral adquirido. Identificaron cinco medidas de participación. Los autores analizaron y criticaron las medidas en relación con el contenido, la validez, la fiabilidad, la utilidad clínica, capacidad de respuesta y fortalezas / debilidades generales. Los autores refieren que no se detectaron estudios que utilizaran análisis factoriales para el LIFE-H en población con estas características, y si bien se destaca la utilidad clínica de este instrumento para evaluar la participación según los criterios propuestos por la CIF, los estudios solo refieren evaluaciones de confiabilidad por medio de múltiples medidas (test-retest, inter-evaluadores) y de validez discriminante y de contenido, pero no de su estructura dimensional (Ziviani et al., 2012).

Así mismo, a nivel clínico, se pueden encontrar estudios en poblaciones que utilizan LIFE-H, justificando la necesidad de generar propiedades psicométricas y pertinencia en la evaluación de la participación en personas que presentan algún grado de discapacidad. La literatura refiere antecedentes en adultos mayores con discapacidad (Desrosiers et al., 2006; Desrosiers et al., 2009; Poulin \& Desrosiers, 2009), pacientes con trastornos neuromusculares (Gagnon, Mathieu, \& Noreau, 2006), adultos mayores con accidentes cerebrovasculares (Desrosiers \& Bourbonnais, 2005; Desrosiers et al., 2006), jóvenes adultos con Síndrome de Down (Foley et al., 2014), mujeres con cáncer (Nikolić et al., 2015) y en niños con parálisis cerebral (Calley et al., 2012; Lepage, Noreau, Bernard, \& Fougeyrollas, 1998; Neves dos Santos, Leticia Pavao, Pererira Santiago, de Fatima Salvini, \& Cicuto Ferreira Rocha, 2013). Estos estudios sustentan la relevancia del LIFE-H en la evaluación establecida por organismos internacionales de la participación en niños, jóvenes y adultos.

En América Latina se han desarrollado algunas investigaciones con la finalidad de adaptar culturalmente este instrumento en pacientes con discapacidad. En Brasil, por ejemplo, se implementó un proceso de adaptación transcultural estandarizado y finalmente una piloto con 10 adultos. Los autores concluyen que la versión final del LIFE-H para su uso en Brasil presentó un grado satisfactorio de equivalencia semántica, idiomática, cultural y conceptual (Nunes de Assumpção et al., 2016).

En Colombia, se realizó una traducción y adaptación cultural del instrumento en una muestra de 6 madres de niños sin condiciones neurológicas, y posteriormente una evaluación de la validez aparente del LIFE-H en su formato para niños, en 19 madres de niños con Parálisis Cerebral por medio de una entrevista cognitiva (Santamaría, Camden, \& Fonseca, 2013). Al igual que el estudio reportado en Brasil, la baja cantidad de participantes y la ausencia de datos psicométricos como confiabilidad o estructura factorial, no permiten conocer las propiedades psicométricas en esta población en Latinoamérica.

En base a lo planteado, la presente investigación tiene como objetivo validar el Life Habits Assessment (LIFE-H) para la evaluación de la participación de niños/as y jóvenes con Parálisis Cerebral en Chile, siendo este un grupo especialmente vulnerable a ser excluido de las actividades e interacciones sociales debido a las características de su enfermedad.

Específicamente se propone a) traducir el formato de joven / adulto; b) evaluar la confiabilidad por consistencia interna del formato joven/ adulto y niños en una muestra de personas con parálisis cerebral y c) comprobar la estructura dimensional del instrumento a través de análisis factorial confirmatorio. Se hipotetizan adecuados niveles de consistencia interna, y una estructura factorial similar a la propuesta por los autores. 


\section{Método}

\section{Diseño}

Se utilizó un diseño instrumental para estudiar las propiedades psicométricas del LIFE-H. Para su ejecución se tomaron en cuenta las recomendaciones que establece la Comisión Internacional de Test (Muñiz, Elosua, \& Hambleton, 2013).

\section{Participantes}

Los participantes fueron reclutados a través de un muestreo no probabilístico, por conveniencia desde escuelas e instituciones de rehabilitación de tres regiones de Chile. El criterio de inclusión fue que los participantes tuvieran diagnóstico de Parálisis Cerebral, con edades entre 9 y 21 años y la habilidad para comprender instrucciones. No se excluyeron participantes por nivel intelectual, funcionalidad motora, modo de comunicación (gestos, vocalizaciones, pictogramas, etc), ni otras características.

La muestra total quedó conformada por 123 personas con parálisis cerebral entre 9 y 21 años de edad $(\mathrm{M}=14.14 ; \mathrm{DE}=2.89)$, de los cuales 73 eran hombres $(59,3 \%)$ y 50 mujeres $(40,7)$. Este grupo fue dividido en niños/as y jóvenes para poder aplicar las dos formas del instrumento LIFE-H. De este modo, el grupo de niños quedó conformado por 57 niños entre nueve y 13 años de edad $(\mathrm{M}=11.44 ; \mathrm{DE}=1.36)$, siendo 34 hombres y 23 mujeres; y 66 jóvenes entre 14 y 21 años de edad $(M=16.47 ; \mathrm{DE}=1.47), 39$ hombres y 27 mujeres. Respecto al tipo específico de parálisis cerebral, el 32,5\% de la muestra tenía hemiplegia, el 17,9\% diplegia, el 19,5\% cuadriplegia y el 30,1\% restante otro tipo o no reportada. No se detectaron diferencias estadísticamente significativas en esta distribución según grupo etario al que pertenecían $\left(\chi^{2}(3)=0,770, p=0,857\right)$.

El tamaño muestral total se alcanzó luego de un período de reclutamiento de aproximadamente 10 meses y tras agotar la pesquisa y solicitud de autorización de los principales centros de rehabilitación y escuelas especiales de la Región del Biobío, Los Lagos y Metropolitana de Chile.

En cuanto a la funcionalidad motora, la muestra incluyó participantes jóvenes y niños/as con rendimiento funcional de nivel I (más capaz) a nivel V (menos capaz) para cada escala. Entre ellos, el 38,2 \% presentaba movilidad sin limitaciones (GMFCS-E \& $\mathrm{R}=\mathrm{I}$ ); $15,5 \%$ caminaba con limitaciones (GMFCS-E \& $\mathrm{R}=\mathrm{II}$ ); $4,9 \%$ se desplazaba usando un dispositivo de movilidad manual (GMFCS-E \& R = III); 2,4\% tenían movilidad autónoma con limitaciones y podían utilizar una silla de ruedas eléctrica (GMFCS-E \& R = IV); y $39 \%$ era transportado en una silla de ruedas manual (GMFCS-E \& R = V). En términos de capacidad de comunicación, 54 participantes $(43,9 \%)$ interactuaba verbalmente; 53 interactuaba con vocalizaciones $(43,1 \%)$; 13 participantes se comunicaban con movimientos de la cabeza (10,6\%); uno con seguimiento de la mirada $(0,8 \%)$; uno con pictogramas $(0,8 \%)$; y uno con signos $(0,8 \%)$.

\section{Instrumento}

Para el presente estudio se empleó la versión 3.0 del Assessment of Life Habits (LIFE-H) (Cuestionario de hábitos de vida LIFE-H) de Fougeyrollas et al., (1998). Este instrumento permite la evaluación de la participación de personas con discapacidad, entendida como el conjunto de actividades cotidianas o hábitos de vida que realiza una persona, resultado de la interacción entre el individuo y los factores del entorno que le rodean. Estas tareas cotidianas se conceptualizan como las actividades diarias y los roles sociales que favorecen o aseguran la supervivencia y el desarrollo de una persona dentro de la sociedad a lo largo de toda su vida (Fougeyrollas et al., 1998). En concreto, se trata de las actividades diarias y los roles sociales reconocidos por las personas o su contexto sociocultural según la edad, el sexo y la identidad socio-cultural.

Este instrumento puede ser respondido tanto por los niños como por sus profesores o padres y posee una versión para niños (5-13 años), y otra para jóvenes y adultos (14-99 años). Ambas poseen casi las mismas preguntas, a excepción de las relacionadas al ámbito del trabajo y la sexualidad.

La versión para niños se compone de 64 ítems y la versión para jóvenes y adultos de 77 ítems, para cada uno de los cuales se debe responder tres preguntas: a) en qué nivel de realización puede ejecutar la actividad el niño/a o joven; b) cuál es el tipo de ayuda que requiere para realizar la actividad y c) cuál es el nivel de satisfacción con 
que logra realizar la actividad. La pregunta por el nivel de realización se responde con una escala Likert de 4 puntos con los conceptos a) Sin dificultad, b) Con dificultad, c) Realizado por sustitución, d) No realizable y e) No aplica; la pregunta por el tipo de ayuda se responde con una escala Likert de 5 puntos con los conceptos a) Sin ayuda, b) Con ayuda técnica, c) Con adaptación o acondicionamiento; d) Con ayuda humana; y la pregunta por la satisfacción con que logra la actividad, con los conceptos a) Muy insatisfecho, b) Insatisfecho, c) Más o menos satisfecho, d) Satisfecho y e) Muy satisfecho.

A partir de las 3 respuestas a cada reactivo, se llega a través de una fórmula, a un puntaje único de 1 a 10 que refleja el grado de participación de la persona en el hábito de vida consultado, donde 1 indica la total restricción en la participación y 10 ninguna restricción.

\section{Procedimiento}

Para la utilización de este instrumento, primeramente se solicitó el permiso editorial a International Network on the Disability Creation Proccess para su adaptación y validación. El formato para niños contaba con una versión en español, pero no el de jóvenes/adultos. Por lo que se procedió a realizar el proceso de traducción de esa versión. Así, el LIFE-H para jóvenes y adultos fue traducido al español por un traductor y la investigadora principal de manera independiente. A continuación, se discutieron ambas versiones y se escogió la mejor traducción para cada ítem. Posteriormente una profesional experta en el idioma español, revisó la versión final respecto a la adecuada estructuración de las frases en el idioma. Posteriormente se empleó la técnica de la entrevista cognitiva para asegurar la adaptación cultural y lingüística del test. El procedimiento contempló la entrevista a nueve docentes que atendían a estudiantes con alguna discapacidad motora, considerando docentes de escuelas regulares y especiales. La realización de la entrevista tomó entre 60 y 90 minutos por cada uno de los entrevistados, posteriormente se realizó un análisis de cada una de las observaciones, corroborando que no existían dificultades en la comprensión de los ítems.

Finalmente, el LIFE-H en sus dos formatos (niños y joven/ adultos) fue aplicado a los terapeutas, profesores o profesionales a cargo de los 123 niños y jóvenes con PC.

Pese a que algunos participantes con PC en la muestra podrían haber sido capaces de responder el LIFE-H por sí mismos, el instrumento en todos los casos fue respondido por su profesor o terapeuta principal, de modo de tener evaluadores equivalentes en la muestra total y evitar el sesgo emanado de combinar diferentes informantes.

Se solicitó el consentimiento informado de los padres o tutores de los participantes y el asentimiento de los participantes se obtuvo en presencia de una tercera persona que fue garante y firmó el documento, asegurando que había expresado su voluntad de participar mediante su método de comunicación habitual (señas, pictograma, vocalizaciones, palabras).

Las aplicaciones fueron realizadas por dos psicólogos entrenados en el uso del instrumento en el lugar de trabajo del profesor o terapeuta en un lugar y fecha de común acuerdo. Esta investigación fue aprobada por el comité de ética de la Universidad de Concepción Chile.

\section{Análisis de datos}

Se utilizaron los programas estadísticos: IBM SPSS STATISTIC (2011, Versión 19) y MPlus 7.1.

Para realizar el análisis de datos se utilizaron los puntajes totales estandarizados en cada indicador de la participación, de acuerdo a la fórmula que contempla el test para convertir los puntajes originales en cada uno de los indicadores a una escala de 1 a 10 puntos. Se realizó de este modo, debido a que por la naturaleza del test, trabajar directamente con todos los ítems resultaba complejo y poco fiable por los datos perdidos que se generan con la opción de respuesta "no aplica", referida a que dicho hábito de vida no está dentro de la cotidianidad del paciente. Así, este tipo de dato faltante no corresponde a un "dato perdido" en el sentido estadístico de la palabra, sino que responde a la imposibilidad de asignar un valor a ese reactivo en particular debido a la situación de vida del evaluado. De este modo, para calcular el nivel de realización del hábito, es preciso calcular la sumatoria de los puntajes brutos y multiplicarlos por $10 \mathrm{y}$ finalmente dividir esto por el número de hábitos que sí aplican a la vida del paciente multiplicados por 9.

Una vez calculado el puntaje estándar en para los 12 indicadores se comparó el puntaje según grupo etario y no se encontraron diferencias estadísticamente significativas en 10 de los 12 indicadores ( $p>0.005)$. El 
único indicador en la que los grupos de niños y jóvenes difirieron fue en "vida comunitaria" $(\mathrm{t}(39,07)=2,33$; $\mathrm{p}=0,025)$. El indicador trabajo, solo considera un ítem en el formato para niños, por lo que hubo un 97,5\% de datos perdidos en este indicador $(n=117)$ y por ende, no se sometió a comparación por grupo etario. Los promedios por indicador para cada uno de los grupos etarios se presentan en la Tabla 1.

Tabla 1. Comparación del puntaje por indicador en cada grupo

\begin{tabular}{|c|c|c|c|c|c|c|c|}
\hline & \multicolumn{2}{|c|}{ Niños/as } & \multicolumn{2}{|r|}{ Jóvenes } & \multirow[b]{2}{*}{ g.l. } & \multirow[b]{2}{*}{$\mathrm{t}$} & \multirow[b]{2}{*}{$\mathrm{P}$} \\
\hline & $\mathrm{n}$ & $\mathrm{M}(\mathrm{SD})$ & $\mathrm{n}$ & $\mathrm{M}(\mathrm{SD})$ & & & \\
\hline Nutrición & 52 & $7.4(2.69)$ & 61 & $7.610(2.49)$ & 103.225 & 0.428 & 0.669 \\
\hline Condición Física & 55 & $6.95(2.44)$ & 62 & $7.310(2.44)$ & 111.365 & 0.797 & 0.427 \\
\hline Cuidado personal & 52 & $5.72(3.13)$ & 62 & $6.230(2.94)$ & 103.898 & 0.891 & 0.375 \\
\hline Comunicación & 54 & $7.1(2.55)$ & 64 & $7.210(2.39)$ & 107.911 & 0.240 & 0.810 \\
\hline Vivienda & 49 & $6.52(3.36)$ & 55 & $7.300(2.77)$ & 91.304 & 1.282 & 0.203 \\
\hline Desplazamiento & 51 & $6.21(3.01)$ & 60 & $6.270(2.94)$ & 103.217 & 0.106 & 0.916 \\
\hline Responsabilidades & 48 & $6.33(3.02)$ & 57 & $6.240(3.05)$ & 98.285 & 0.152 & 0.880 \\
\hline Relaciones interpersonales & 53 & $8.43(2.07)$ & 62 & $8.520(2.13)$ & 109.098 & 0.229 & 0.819 \\
\hline Vida en Comunidad & 22 & $7.58(2.82)$ & 55 & $5.890(3.00)$ & 39.072 & 2.332 & 0.025 \\
\hline Educación & 53 & $6.46(2.6)$ & 63 & $6.680(2.67)$ & 109.615 & 0.448 & 0.655 \\
\hline Trabajo & 3 & $6.67(5.77)$ & 53 & $6.930(2.70)$ & 0.050 & 0.078 & -- \\
\hline Recreación & 50 & $6.63(2.93)$ & 51 & $5.820(2.98)$ & 96.997 & 1.377 & 0.172 \\
\hline
\end{tabular}

Debido a que 10 de 12 indicadores no diferían significativamente en sus promedios, se procedió a juntar las muestras para posibilitar la realización del análisis factorial confirmatorio y alcanzar el número requerido de 10 personas por ítem.

A continuación, se realizó una imputación de datos perdidos con el algoritmo de EM (esperanza-maximización) para poder trabajar con la totalidad de los datos. La prueba de Kolmogorov-Smirnov corroboró la distribución normal de las puntuaciones. Luego se realizó un análisis descriptivo de los datos y de consistencia interna mediante el coeficiente alfa de Cronbach, seguido de un análisis factorial confirmatorio con el método de máxima verosimilitud.

Para el análisis factorial confirmatorio se emplearon diversos tipos de ajustes que fueron seleccionados sobre la base de rendimiento satisfactorio en otros estudios psicométricos (Brown, 2014). Se empleó los índices de Chi-cuadrado; donde se espera encontrar valores no significativos. La raíz cuadrada media de error de aproximación (RMSEA) considera un buen ajuste si presenta valores menores a .05-.08 y un intervalo de confianza al $90 \%$ (I.C.) entre 0 y .05; el índice de ajuste comparativo (CFI) y índice de Tucker-Lewis (TLI) fueron generados esperando valores superiores a .95 (Brown, 2014; Geiser, 2012; Hair, Rolph,Tatham, Ronland, Black \& William,1999).

Seguidamente, debido a que no se comprobó la estructura de dos dimensiones esperada, se realizó un análisis factorial exploratorio bajo el método de extracción de máxima verosimilitud. Para determinar la cantidad de factores, se analizaron los autovalores (Eigen) y el gráfico de sedimentación de Cattell (Scree Plot) y para identificar que ítems formaban cada componente se analizaron las cargas factoriales asumiendo como adecuado cargas iguales o mayores a .40 (Norman \& Streiner, 2008). 


\section{Resultados}

Los resultados del estudio fueron organizados en dos apartados, el primero se presentan los estadísticos descriptivos obtenidos en la muestra y segundo se describe los análisis de las propiedades psicométricas del LIFE-H.

\section{Resultados descriptivos}

En la Tabla 2 se presentan las medidas de tendencia central y de dispersión obtenidas en la muestra de participantes con Parálisis Cerebral. Los resultados evidencian que el indicador Cuidado Personal es la que posee menores puntajes por parte de los niños y jóvenes. En tal sentido, las respuestas de los niños y jóvenes refieren mayores interrupciones en la realización de actividades de la higiene personal de forma independiente. El resto de los indicadores presentan mayores puntuaciones, siendo la nutrición el indicador que más destaca, indicando que los niños y jóvenes tienen mayor desarrollo en este ámbito en comparación a las otras dimensiones.

Con respecto a la distribución de las respuestas de los participantes, la asimetría y la curtosis reportan valores dentro del marco esperado (entre -2 y 2) confirmando la utilización del método de máxima verisimilitud en los análisis de la validez a partir de los análisis factoriales (Lloret-Segura, Ferreres-Traver, Hernández-Baeza, \& Tomás-Marco, 2014).

Tabla 2. Estadísticas descriptivas de los indicadores de LIFE-H

\begin{tabular}{lrrrrr}
\hline & Mínimo & Máximo & Media(DS) & Asimetría & \multicolumn{1}{c}{ Curtosis } \\
\hline Nutrición & 0 & 10 & $7.52(2.504)$ & -.798 & -.244 \\
Condición Física & 2 & 10 & $7.10(2.401)$ & -.558 & -.644 \\
Cuidado Personal & 0 & 10 & $5.97(3.003)$ & -.225 & -1.106 \\
Comunicación & 1 & 10 & $7.18(2.409)$ & -.594 & -.752 \\
Vivienda & 0 & 10 & $6.78(3.077)$ & -.645 & -.786 \\
Desplazamiento & 1 & 10 & $6.13(2.892)$ & .009 & -1.319 \\
Responsabilidades & 2 & 10 & $7.19(2.740)$ & -.521 & -1.217 \\
Relaciones Interpersonales & 1 & 10 & $7.47(2.701)$ & -.748 & -.731 \\
Vida en Comunidad & 1 & 10 & $6.42(2.839)$ & -.020 & -1.388 \\
Educación & 1 & 10 & $6.55(2.595)$ & -.174 & -1.260 \\
Trabajo & 0 & 10 & $6.10(3.014)$ & -.320 & -1.228 \\
Recreación & 0 & 10 & $6.15(2.816)$ & .030 & -1.268 \\
\hline
\end{tabular}

Nota: $\mathrm{N}=120$

\section{Análisis Psicométrico}

\section{CONFIAbILIDAD POR CONSISTENCIA INTERNA}

Para el análisis de la confiabilidad de las respuestas obtenidas por medio de LIFE-H en personas con parálisis cerebral, se utilizaron los índices de Alpha de Cronbach y Omega de Mc Donald. Estos índices deben presentar valores superiores a .70 para considerarse como adecuados (Luján-Tangarife \& Cardona-Arias, 2015). En este caso, los resultados descritos en la Tabla 3 muestran altos niveles de confiabilidad para cada dimensión propuesta por los autores: Hábitos de vida y Roles Sociales. 
Tabla 3. Índice de Confiabilidad para las dimensiones del LIFE-H

\begin{tabular}{|c|c|c|c|c|}
\hline & & $\begin{array}{c}\text { Correlación ítem-total } \\
\text { corregida }\end{array}$ & $\begin{array}{c}\text { Alfa de } \\
\text { Cronbach }\end{array}$ & Omega \\
\hline \multirow[t]{7}{*}{ Hábitos de Vida $(\mathrm{n}=6)$} & & & 0.938 & 0.940 \\
\hline & Nutrición & 0.799 & & \\
\hline & Condición Física & 0.855 & & \\
\hline & Cuidado Personal & 0.893 & & \\
\hline & Comunicación & 0.772 & & \\
\hline & Vivienda & 0.840 & & \\
\hline & Desplazamiento & 0.759 & & \\
\hline \multirow[t]{7}{*}{ Roles Sociales $(n=6)$} & & & 0.898 & 0.903 \\
\hline & Responsabilidades & 0.582 & & \\
\hline & Relaciones Interpersonales & 0.550 & & \\
\hline & Vida en Comunidad & 0.775 & & \\
\hline & Educación & 0.853 & & \\
\hline & Trabajo & 0.788 & & \\
\hline & Recreación & 0.819 & & \\
\hline
\end{tabular}

\section{Análisis Factorial Confirmatorio}

Se realizó un análisis factorial confirmatorio para analizar la validez del LIFE-H y poner a prueba la propuesta teórica original de una estructura de dos factores (Hábitos y Roles Sociales) construidos por 6 indicadores cada uno. Se empleó el método de estimación de máxima verosimilitud y se calcularon varios índices de bondad de ajuste para determinar la estructura propuesta. Los resultados descritos en la Tabla 4, muestran que las medidas de bondad de ajuste calculadas para este modelo no fueron satisfactorias. Se presenta un valor de chi cuadrado significativo, el error medio cuadrático (RMSEA) es superior al nivel considerado como adecuado (.05-.08) y el índice de ajuste comparativo (CFI) y el Índice Tucker-Lewis (TLI) no cumplen con los rangos aceptados (Brown, 2014; Geiser, 2012).

Tabla 4. Índices del Análisis Factorial Confirmatorio del LIFE-H.

\begin{tabular}{clllll}
\hline $\mathrm{X}^{2}$ & $\mathrm{gl}$ & RMSEA & $90 \%$ CI & CFI & TLI \\
\hline 18.773 & 53 & 0.159 & $0.138-0.182$ & 0.840 & 0.800 \\
\hline
\end{tabular}

Nota: $\mathrm{N}=123 ; \mathrm{df}=$ grados de libertad del modelo; RMSEA= Error Medio Cuadrático de Aproximación; $(90 \%$ CI)= Intervalo de confianza del 90\% para RMSEA; CFI=Índice de Ajuste Comparativo; TLI=Índice Tucker-Lewis; ${ }^{\mathrm{p}}<.01$.

En vista de los resultados obtenidos, se decidió realizar un análisis factorial exploratorio, para evaluar la estructura dimensional del LIFE-H en Chile. Se utilizó el método de extracción de máxima verosimilitud, dada la normal distribución de los datos. 
El estadístico de adecuación muestral de Kayser-Meyer-Olkin (KMO) el cual fue de .847, el test de esfericidad de Bartlett fue significativo $\left(\mathrm{X}^{2}(66)=1603.89 ; \mathrm{p}<.001\right)$, confirman la existencia de correlaciones suficientes para generar el análisis de factores (Norman \& Streiner, 2008). Los resultados mostraron una escala unifactorial con un autovalor (Eigen) de 8.31, que explicaba el $69.55 \%$ de la varianza. El gráfico de sedimentación de Cattell "Scree Plot" confirmó valores concentrados en un solo factor

Las cargas factoriales de los ítems alcanzaron valores entre 0.59 y 0.93 , dando cumplimiento a los estándares teóricos para mantener la estructura factorial igual que el instrumento original (ver Tabla 5).

Tabla 5. Cargas factoriales de los indicadores del LIFE-H

\begin{tabular}{lr}
\hline Indicadores & Cargas factoriales \\
\hline Nutrición & .813 \\
Condición Física & .869 \\
Cuidado Personal & .933 \\
Comunicación & .773 \\
Vivienda & .889 \\
Desplazamiento & .815 \\
Responsabilidades & .590 \\
Relaciones Interpersonales & .664 \\
Vida en Comunidad & .787 \\
Educación & .883 \\
Trabajo & .874 \\
Recreación & .860 \\
\hline
\end{tabular}

\section{Discusión}

Esta investigación tuvo como objetivo validar el Life Habits Assessment (LIFE-H) para la evaluación de la participación de niños/as y jóvenes con Parálisis Cerebral y de esta manera aportar con el conocimiento de las propiedades psicométricas de un instrumento útil para la evaluación de este constructo en población con condiciones neurológicas y la planificación de la rehabilitación desde la mirada propuesta por la CIF.

Se presentan los procedimientos realizados para garantizar la equivalencia cultural y lingüística del LIFE-H con su versión original, así como análisis que apoyan sus propiedades psicométricas para este contexto. Los resultados obtenidos en el presente estudio permiten afirmar que el cuestionario de evaluación de los hábitos de vida (LIFE-H), es un instrumento válido y confiable para el estudio de la participación en niños y jóvenes chilenos, pero entendiendo la participación como un constructo global.

En los procesos de validación de escalas es aconsejable el uso de estudios pilotos para corroborar la adaptación lingüística y cultural de los instrumentos (Ramada-Rodilla, Serra-Pujadas, \& Delclós-Clanchet, 2013), debido a tipo de participantes del estudio y la dificultad para el acceso a grandes personas con estas características, se decidió utilizar a la entrevista cognitiva, como una herramienta que permite asegurarse de que los ítems e instrucciones son correctamente comprendidos, así como recoger información sobre posibles errores de contenido o formato que se pueden corregir antes de pasar a la fase operacional y conocer las reacciones de las personas que realizan la prueba.

En lo referido a la validez, los autores decidimos inicialmente evaluar este aspecto por medio del análisis factorial confirmatorio, con la finalidad de "confirmar" la teoría propuesta por los creadores de la escala tal y como es recomendado teóricamente (Lloret-Segura et al., 2014), sin embargo los resultados encontrados no fueron satisfactorios desde el punto de vista estadístico. En tal sentido y por la poca evidencia en el uso del análisis 
factorial como una herramienta para la evaluación de la validez del LIFE-H, se decidió emplear el análisis factorial exploratorio con la finalidad de identificar los factores latentes que subyacen al constructo de participación.

El análisis factorial mostró que el LIFE-H es un instrumento con un factor general que incluye los 12 indicadores relacionados con la vida diaria y los roles desempeñados en ella. A diferencia de la propuesta original donde las actividades están dividas en dos grandes dominios de actividades de vida diaria y roles (Fougeyrollas et al., 1998), esto puede deberse a que la participación conceptualmente se sustenta bajo un modelo integrador donde los dominios de la vida y la enfermedad se encuentran en interacción reciproca constante (Clasificación Internacional del Funcionamiento, de la discapacidad y de la salud, 2001). También puede deberse a que la comprensión de los comportamientos esperados y que se encuentran asociados al constructo difieren entre los grupos culturales (Chahín-Pinzón, 2014). Es decir que en este caso la participación no está determinada por dos dimensiones (actividades de vida diaria y roles), como lo plantean en el diseño original, sino que corresponden a un único factor que integra todas las construcciones que conforman la escala.

El LIFE-H es un instrumento con adecuados índices de confiabilidad, siendo estos superiores a los obtenidos en la propiedades psicométricas originales (versión abreviada $\alpha \geq .82$; versión original $\alpha \geq .90$ ) (Fougeyrollas et al., 1998) y en estudios posteriores en niños con diagnóstico de parálisis cerebral.

En futuras investigaciones sería interesante complementar los análisis realizados con la evaluación de otras propiedades del LIFE-H en este contexto, tales como la fiabilidad test-retest, la validez de criterio. Así como la generación de propiedades psicométricas en niños y jóvenes con y sin discapacidad, con la finalidad de realizar estudios comparativos que incremente la comprensión de la participación infanto-juvenil en el contexto chileno.

Esta investigación presenta ciertas fortalezas en su desarrollo, este es el primer estudio que busca adaptar el LIFE-H a una muestra de niños y jóvenes con Parálisis Cerebral chilenos, en tal sentido sus aportes proporcionan una base para el estudio de la participación en este contexto, lo que beneficiaria en términos prácticos a las evaluaciones realizada por los profesionales de salud. Como conclusión, en este estudio se proporcionan evidencias de que el LIFE-H puede utilizarse como una medida valida y confiable de la participación en niños y jóvenes con parálisis cerebral. A lo anterior se une que es un instrumento sencillo y flexible para ser completado, siendo una característica es relevante para su uso en contextos de salud.

\section{Referencias}

Anaby, D., \& Law, M. (2013). The role of participation in the lives of children and young people with neurological and developmental conditions. Life Quality Outcomes in Children and Young People with Neurological and Developmental Conditions; Ronen, G., Rosenbaum, P., Eds, 51-64.

Brown, T. A. (2014). Confirmatory factor analysis for applied research (Second ed.). New York: Guilford Publications.

Calley, A., Williams, S., Reid, S., Blair, E., Valentine, J., Girdler, S., \& Elliott, C. (2012). A comparison of activity, participation and quality of life in children with and without spastic diplegia cerebral palsy. Disability and rehabilitation, 34, 1306-1310.

Chahín-Pinzón, N. (2014). Aspectos a tener en cuenta cuando se realiza una adaptación de test entre diferentes culturas. Psychologia. Avances de la disciplina, 8, 109-112.

Chien, C.-W., Rodger, S., Copley, J., \& Skorka, K. (2014). Comparative content review of children's participation measures using the international classification of functioning, disability and health-children and youth. Archives of physical medicine and rehabilitation, 95, 141-152.

Desrosiers, J., \& Bourbonnais, D. (2005). Participation after stroke compared to normal aging. J Rehabil Med, 37, $353-357$.

Desrosiers, J., Noreau, L., Rochette, A., Bourbonnais, D., Bravo, G., \& Bourget, A. (2006). Predictors of long-term participation after stroke. Disability and rehabilitation, 28, 221-230.

Desrosiers, J., Robichaud, L., Demers, L., Gélinas, I., Noreau, L., \& Durand, D. (2009). Comparison and correlates of participation in older adults without disabilities. Archives of Gerontology and Geriatrics, 49, 397-403.

Figueiredo, S., Korner-Bitensky, N., Rochette, A., \& Desrosiers, J. (2010). Use of the LIFE-H in stroke rehabilitation: A structured review of its psychometric properties. Disability and Rehabilitation, 32, 705-712. doi:10.3109/09638280903295458

Foley, K.-R., Girdler, S., Bourke, J., Jacoby, P., Llewellyn, G., Einfeld, S., . . Leonard, H. (2014). Influence of the environment on participation in social roles for young adults with down syndrome. PloS one, 9, e108413. 
Fougeyrollas, P., Noreau, L., Bergeron, H., Cloutier, R., Dion, S., \& St-Michel, G. (1998). Social consequences of long term impairments and disabilities: conceptual approach and assessment of handicap. International journal of rehabilitation research. Internationale Zeitschrift fur Rehabilitationsforschung. Revue internationale de recherches de readaptation, 21, 127-141.

Gagnon, C., Mathieu, J., \& Noreau, L. (2006). Measurement of participation in myotonic dystrophy: reliability of the LIFE-H. Neuromuscular Disorders, 16, 262-268.

Geiser, C. (2012). Data analysis with Mplus: Guilford Press.

Hair, J. F. A., Rolph E., Tatham, Ronland L., Black, William C. (1999). Análisis multivariante (5th ed.). Madrid, España: Prentice Hall.

Lepage, C., Noreau, L., Bernard, P., \& Fougeyrollas, P. (1998). Profile of handicap situations in children with cerebral palsy. Scandinavian Journal of Rehabilitation Medicine, 30, 263-272.

Levasseur, M., Desrosiers, J., \& Noreau, L. (2004). Is social participation associated with quality of life of older adults with physical disabilities? Disability and Rehabilitation, 26, 1206-1213. doi:10.1080/09638280412331270371

Levasseur, M., Desrosiers, J., \& St-Cyr Tribble, D. (2008). Subjective Quality-of-Life Predictors for Older Adults with Physical Disabilities. American Journal of Physical Medicine \& Rehabilitation, 87, 830-841. doi:10.1097/PHM.0b013e318186b5bd

Lloret-Segura, S., Ferreres-Traver, A., Hernández-Baeza, A., \& Tomás-Marco, I. (2014). El análisis factorial exploratorio de los ítems: una guía práctica, revisada y actualizada. Anales de Psicología, 30, 1151-1169.

Luján-Tangarife, J., \& Cardona-Arias, J. (2015). Construcción y validación de escalas de medición en salud: revisión de propiedades psicométricas. Archivos de Medicina, 11.

Michelsen, S., Flachs, E., Damsgaard, M., Parkes, J., Parkinson, K., Rapp, M., . . Uldall, P. (2014). European study of frequency of participation of adolescents with and without cerebral palsy. European Journal of Paediatric Neurology, 18, 282-294. doi:https:// doi.org/10.1016/j.ejpn.2013.12.003

Michelsen, S. I., Uldall, P., Kejs, A. M. T., \& Madsen, M. (2005). Education and employment prospects in cerebral palsy. Developmental medicine and child neurology, 47, 511-517.

Morris. (2007). Measuring children's participation. Developmental Medicine \& Child Neurology, 49, 645-645. doi:10.1111/j.14698749.2007.00645.x

Morris, C., Kurinczuk, J., \& Fitzpatrick, R. (2005). Child or family assessed measures of activity performance and participation for children with cerebral palsy: a structured review. Child: Care, Health and Development, 31, 397-407. doi:10.1111/j.1365-2214.2005.00519.x

Muñiz, J., Elosua, P., \& Hambleton, R. K. (2013). Directrices para la traducción y adaptación de los tests: segunda edición. Psicothema, 25, 151-157.

Nahuelhual, P., Giaconi, C., \& Machuca, A. (2018). Medición de la participación en niños y adolescentes con y sin discapacidad: una revisión sistemática. Revista Chilena de Pediatría, 88.

Neves dos Santos, A., Leticia Pavao, S., Pererira Santiago, P. R., de Fatima Salvini, T., \& Cicuto Ferreira Rocha, N. A. (2013). Sit-to-stand movement in children with hemiplegic cerebral palsy: relationship with knee extensor torque and social participation. Research in developmental disabilities, 34, 2023-2032.

Nikolić, S., Ilić-Stošović, D., Kolarević, I., Đurđević, A., Ilić, S., \& Đuričić, M. (2015). Social participation of women with breast cancer. Vojnosanitetski pregled, 72, 148-154.

Noreau, L., Desrosiers, J., Robichaud, L., Fougeyrollas, P., Rochette, A., \& Viscogliosi, C. (2004). Measuring social participation: reliability of the LIFE-H in older adults with disabilities. Disability and Rehabilitation, 26, 346-352. doi:10.1080/09638280410001658649

Noreau, L., Lepage, C., Boissiere, L., Picard, R., Fougeyrollas, P., Mathieu, J., . . Nadeau, L. (2007). Measuring participation in children with disabilities using the Assessment of Life Habits. Developmental Medicine \& Child Neurology, 49, 666-671.

Norman, G. R., \& Streiner, D. L. (2008). Biostatistics: the bare essentials.

Nunes de Assumpção, F. S., de Faria-Fortini, I., Lopes Basílio, M., Magalhães, d. C., Cesinando de Carvalho, A., \& Teixeira-Salmela, L. F. (2016). Adaptação transcultural do LIFE-H 3.1: um instrumento de avaliação da participação social. Cadernos de Saúde Pública, $32, \mathrm{e} 00061015$.

Organización Mundial de la Salud, (2001). Clasificación Internacional del Funcionamiento, de la discapacidad y de la salud. Madrid, España.

Piskur, B., Daniels, R., Jongmans, M. J., Ketelaar, M., Smeets, R., Norton, M., \& Beurskens, A. (2014). Participation and social participation: are they distinct concepts? Clinical Rehabilitation, 28, 211-220. doi:10.1177/0269215513499029

Poulin, V., \& Desrosiers, J. (2009). Reliability of the LIFE-H satisfaction scale and relationship between participation and satisfaction of older adults with disabilities. Disability and Rehabilitation, 31, 1311-1317. doi:10.1080/09638280802572957

Ramada-Rodilla, J. M., Serra-Pujadas, C., \& Delclós-Clanchet, G. L. (2013). Adaptación cultural y validación de cuestionarios de salud: revisión y recomendaciones metodológicas. Salud pública de México, 55, 57-66.

Ziviani, J., Desha, L., Feeney, R., \& Boyd, R. (2012). Measures of Participation Outcomes and Environmental Considerations for Children With Acquired Brain Injury: A Systematic Review. Brain Impairment, 11, 93-112. doi:10.1375/brim.11.2.93 